\title{
Chinese Expert Consensus on Critical Care Ultrasound Applications at COVID-19 Pandemic
}

\author{
Faqin Lv, MD ${ }^{a}$, Jinrui Wang, MD ${ }^{b, *}$, Xing Yu, MD ${ }^{c, *}$, Aiping Yang, MD ${ }^{d, *}$, Ji-Bin Liu, MD ${ }^{e, *}$, Linxue Qian, MD ${ }^{f}$, \\ Huixiong Xu, MD ${ }^{g}$, Ligang Cui, MD ${ }^{b}$, Mingxing Xie, MD ${ }^{h}$, Xi Liu, MD ${ }^{i}$, Chengzhong Peng, MD ${ }^{j}$, Yi Huang, MD ${ }^{k}$, \\ Haiyan Kou, MD ${ }^{l}$, Shengzheng $W u, M D^{a}$, Xi Yang, MD ${ }^{m}$, Bin Tu, MD ${ }^{n}$; Huaping Jia, MD ${ }^{o}$, Qingyi Meng, MD ${ }^{p}$, \\ Jie Liu, MD ${ }^{a}$, Ruizhong Ye, MD ${ }^{j}$
}

\begin{abstract}
${ }^{a}$ Hainan Hospital of PLA General Hospital, Sanya, China; ${ }^{b}$ Peking University Third Hospital, Beijing, China; ${ }^{c}$ National Health Commission Contraceptives Management Center for Health, Beijing, China; ${ }^{d}$ National Health Commission Capacity Building and Continuing Education Center, Beijing, China; ${ }^{e}$ Thomas Jefferson University, Philadelphia, USA; ${ }^{f}$ Beijing Friendship Hospital, Capital Medical University, Beijing, China; ${ }^{g}$ Shanghai Tenth People's Hospital, Tongji University, Shanghai, China; ${ }^{h}$ Union Hospital of Tongji Medical College, Huazhong University of Science and Technology, Wuhan, China; ${ }^{i}$ Air Force Medical Center, Beijing, China, ${ }^{j}$ Zhejiang Provincial People's Hospital, Hangzhou, China; ${ }^{k}$ Xi'an Chest Hospital, Xi'an, China; ${ }^{\prime}$ The Third Medical Center of PLA General Hospital, Beijing, China; ${ }^{m}$ Hanyang Hospital Affiliated to Wuhan University of Science and Technology, Wuhan, China; ${ }^{n}$ Foshan Hospital of Traditional Chinese Medicine, Foshan, China; ${ }^{o}$ PLA Strategic Support Force Characteristic Medical Center, Beijing, China;

${ }^{p}$ The First Medical Center of PLA General Hospital, Beijing, China
\end{abstract}

Received April 01, 2020; revision received April 03, 2020; accepted April 10, 2020

\begin{abstract}
Summary: The spread of new coronavirus (SARS-Cov-2) follows a different pattern than previous respiratory viruses, posing a serious public health risk worldwide. World Health Organization (WHO) named the disease as COVID-19 and declared it a pandemic. COVID-19 is characterized by highly contagious nature, rapid transmission, swift clinical course, profound worldwide impact, and high mortality among critically ill patients. Chest X-ray, computerized tomography (CT), and ultrasound are commonly used imaging modalities. Among them, ultrasound, due to its portability and non-invasiveness, can be easily moved to the bedside for examination at any time. In addition, with use of $4 \mathrm{G}$ or $5 \mathrm{G}$ networks, remote ultrasound consultation can also be performed, which allows ultrasound to be used in isolated medial areas. Besides, the contact surface of ultrasound probe with patients is small and easy to be disinfected. Therefore, ultrasound has gotten lots of positive feedbacks from the frontline healthcare workers, and it has played an indispensable role in the course of COVID-19 diagnosis and follow up.
\end{abstract}

Key words: COVID-19; Lung; Cardiac; Ultrasound; Bedside assessment; Remote ultrasound

Advanced Ultrasound in Diagnosis and Therapy 2020;02:027-042

DOI: $10.37015 / A U D T .2020 .200029$

\section{Background of COVID-19}

A new coronavirus (SARS-Cov-2) has infected more than 1000,000 people worldwide by April of 2020 , with more than $10 \%$ of patients developed severe disease [1]. SARS-Cov-2 has an average diameter of about $120 \mathrm{~nm}$, while the size of bacteria is measured in micrometers. The virus particles enter the airways and lungs by breathing, and it could reach the bronchioles and alveoli. Therefore, SARS-Cov-2 mostly distributed in the terminal alveoli under the pleura [2]. The autopsy

\footnotetext{
* Corresponding authors: Jinrui Wang, MD, Department of Ultrasound, Peking University Third Hospital, China. e-mail: jinrui wang@sina.com; Xing Yu, MD, National Health Commission Contraceptives Management Center for Health, Beijing, China. e-mail: 13901205223@163.com; Aiping Yang, MD, National Health Commission Capacity Building and Continuing Education Center, Beijing, China.e-mail: yap1341@sina.com; Ji-Bin Liu, MD, 796 Main Building, 132 South 10 ${ }^{\text {th }}$ Street, Philadelphia, USA. e-mail: ji-bin.liu@ jefferson.edu
} unrestricted use, distribution and reproduction in any medium provided that the original work is properly attributed. 
and pathology of COVID-19 lung tissue showed diffuse lesions of the alveoli with mucus exudation, which caused consolidation of the lungs [3]. On CT, groundglass opacities appear on the periphery of the diseased lungs or near the pleura, but pleural effusion is rare [4]. Ultrasound has been shown a great imaging modality to show the distribution of COVID-19 lesions [5-7].

From a clinical point of view, the total number of peripheral white blood cells in the early stage of the disease is normal or decreased with low absolute lymphocyte count, indicating a large number of lymphocytes are consumed. Thus, patients' immunity system will be weakened, and bacterial infection can easily occur. Some patients would develop refractory hypoxemia. Viral infection can also cause damage to other organs throughout the body, such as heart and kidneys, further resulting in decreased heart rate and poor resuscitation outcomes.

\section{Role of Ultrasound for Evaluation of COVID-19}

\section{Basic concepts and lung ultrasound imaging}

Lung volume is determined by the volume of gas in the lungs at a given time during the respiratory cycle. The gas in the lung create a huge acoustic impedance difference to differentiate between lung tissue and the pleura, which covering the lung surface. Therefore, ultrasound waves will be reflected when it reaches the pleura, and imaging will only show the pleural layer in healthy lungs. However, when normal air-to-liquid ratio of the lungs is changed by specific lung diseases, especially when the pleura gets involved, ultrasound waves can be reflected locally to form unique ultrasound images to help locate the lung lesions [8].

\subsection{Acoustic impedance difference}

When the interface is larger than the wavelength and the acoustic impedance difference is greater than one thousandth $(1 \%)$, the echo reflection will occur, which is the basis of ultrasound imaging. The strength of the reflected wave is determined by the difference in acoustic impedance between the two media. The larger the difference in acoustic impedance, the stronger the reflection (Fig. 1).

\subsection{Multiple reflections and reverberation effects}

Between two parallel reflection interfaces, sound waves can be reflected back and forth multiple times and received by the probe. Ultrasonic machine can record these multiple reflected echoes, called multiple reflections, or reverb effect. The first reflected echo is an objective position, and then the time intervals between two echoes will be gradually increased, and so does the distance. This is related to the A-lines in lung ultrasound (Fig. 2).
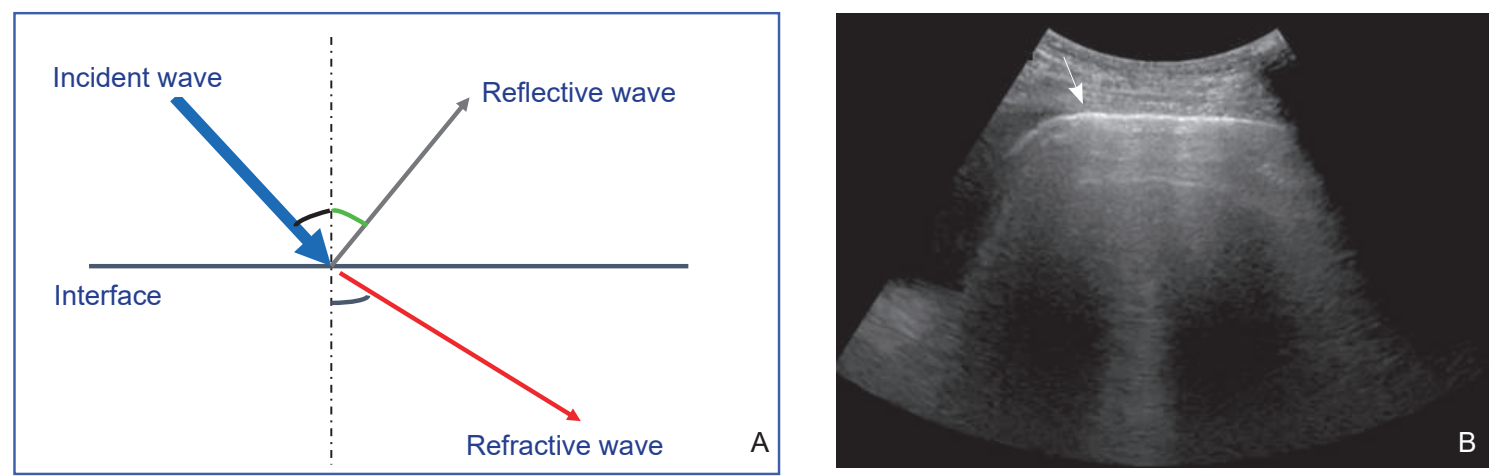

Figure 1 Acoustic impedance difference. (A) Schematic diagram of echo reflection that occurs when the interface is larger than the wavelength; (B) Pleural lines appear as strong echoes in grey-scale ultrasound image (white arrow).

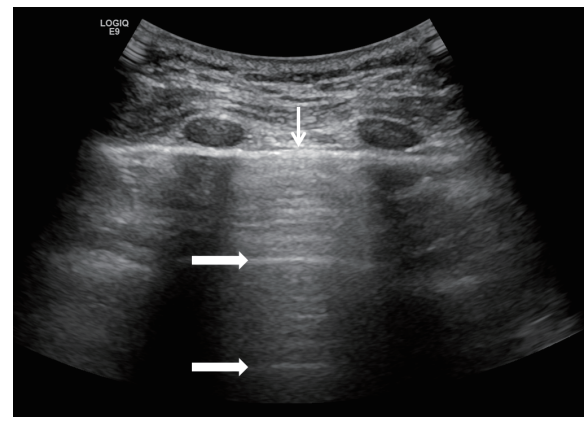

Figure 2 A-line. The two-dimensional ultrasound image parallel to the pleural line are the A-line (2 thick arrows) and pleural line (thin arrow).

\subsection{Comet tail sign}

When a sound beam propagating in soft tissue encounters a thin layer of strong reflection interface such as water bubbles or small crystals, ultrasound can generate multiple reflections in the interior until attenuation, and the reflected echo is received by the probe to produce an internal reverberation. The "comet tail sign" is shown in the sonogram (Fig. 3).

\subsection{Ringing artifacts}

Ultrasonic waves generate strong vibrations between 

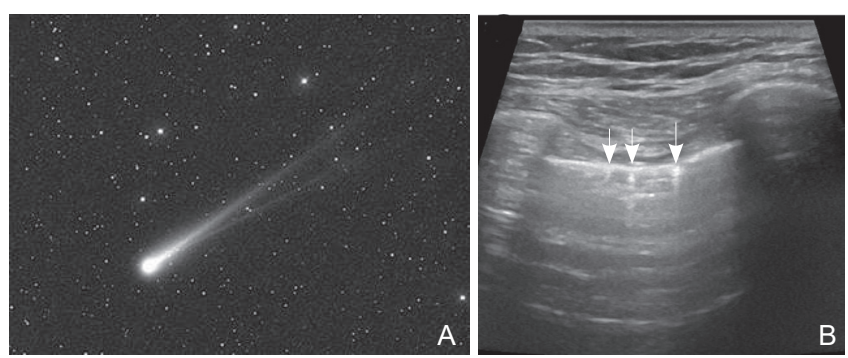

Figure 3 Comet tail sign. (A) Image of a comet flashing in the air with a tail; (B) Comet tail sign (arrow) generated by the gas on the lung surface in a two-dimensional ultrasound image.

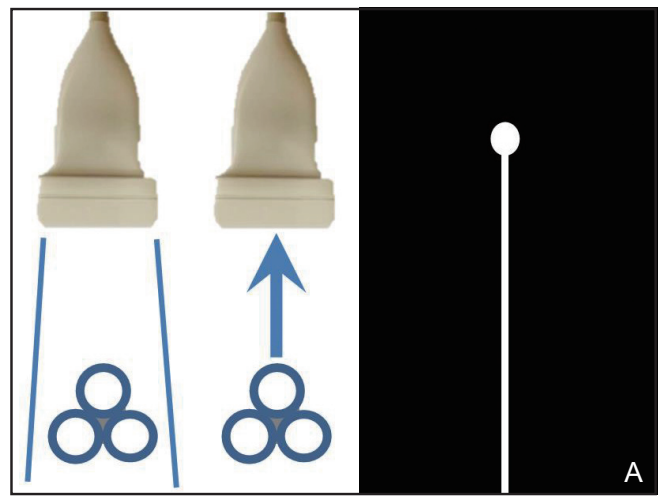

liquids surrounded by gas, generate continuous echoes and are received by the probe. It is not attenuated at the rear, like a laser, such as the B-lines in lung ultrasound (Fig. 4).

\subsection{M-mode ultrasound}

M-mode ultrasound uses a single beam scanning method to make the M-type sampling line pass through human tissues in sequence, and the curve formed by the echo of the tissue passing by the sampling line over time is displayed in an image. It is important in pulmonary and cardiac ultrasound assessment (Fig. 5).

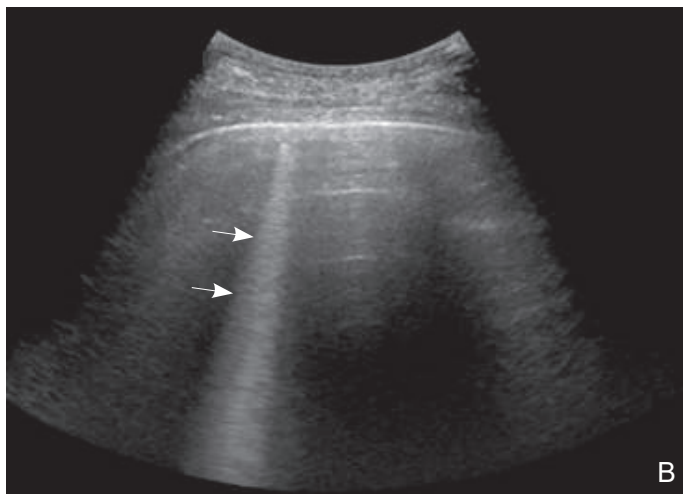

Figure 4 Ringing artifacts. (A) Schematic diagram of ultrasonic waves generating strong oscillations between gas-wrapped liquids, which generate continuous echoes and are received by the probe; (B) Bubbles in the alveoli are oscillated and received by the probe under ultrasound excitation, and the acoustic image is formed with the pleura line and laser beam-like strong echo, B-line (arrow).
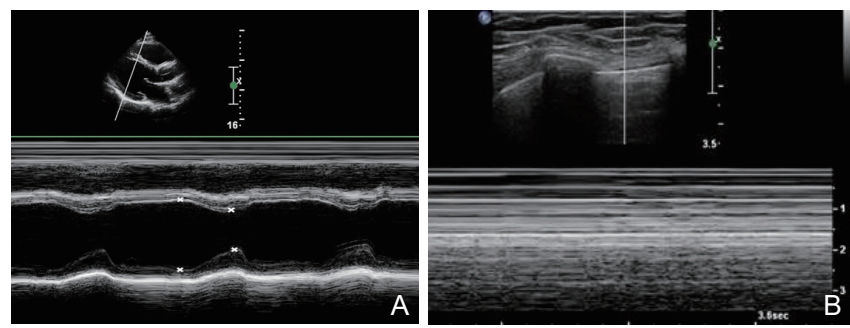

Figure 5 M-mode ultrasound image. (A) M-mode echocardiogram of the heart; (B) M-mode echogram of normal lung. The vertical axis (Y axis) represents distance; the horizontal axis ( $\mathrm{X}$ axis) represents time. The echoes of different depths are expanded with time to form a time-motion curve.

Expert opinion: The content of the article refers to the literature on the principles of pulmonary ultrasound imaging, and combines the clinical practical experience of COVID-19 to explain important physical concepts of pulmonary ultrasound. (Strength of recommendation: B. Recommendation. Positive evidence-based evidence can increase awareness of pulmonary ultrasound applications.)

\section{Ultrasound Equipment and Its Adjustment}

\subsection{Ultrasound equipment}

Since COVID-19 is a highly contagious disease, it is reasonable to use portable ultrasound, palm ultrasound, robotic ultrasound, and remote radiology techniques in hospitals near epidemic area when necessary.

\subsection{Selection of probes and examination conditions}

The ultrasound examination is mainly focusing on heart and lungs. For critically ill patients with potential multiple organs failure, liver, kidney and other important intraabdominal organs, and vascular structures should also be examined. In addition, a comprehensive ultrasound evaluation also plays an important role for monitoring of lower extremity deep venous thrombosis and the placement of catheters in patients who have sever disease. Therefore, ultrasound examination on COVID-19 patients requires the application of phased array, convex array, linear array, and other types of probes, to thoroughly evaluate heart, abdomen, blood vessels, and superficial organs.

\subsection{Image storage and analysis}

In order to minimize exposure, ultrasound providers should follow the institutional protocol to quickly and comprehensively collect dynamic and static image data, and wait until leaving the contaminated area for further in-depth data analysis.

Expert opinion: The content of the clause refers to domestic and foreign literature, and combines clinical practice experience, and prompts the factors affecting 
ultrasound diagnosis such as equipment and imaging parameter conditions. (Recommended strength: A. strongly recommended: Positive evidence-based evidence can improve ultrasound diagnosis and clinical evaluation and reduce missed diagnosis and misdiagnosis.)

\section{Ultrasound Evaluation of COVID-19}

\subsection{Normal pulmonary ultrasound signs \\ 3.3.1 Pleural line}

When the probe scans the lung surface vertically, the sound beam will produce a strong echo formed by a large number of reflections at the interface between the chest wall and the visceral pleura-lung surface.

\subsubsection{A-line}

Also called horizontal line, can be recognized as evenly spaced, parallel lines with gradually decreasing echogenicity. See Figure 3.

\subsubsection{Bat sign}

The probe scans along the longitudinal section of the human body, showing the lung between the two adjacent ribs. The association of ribs and hyperechoic intercostal pleural line make a solid landmark called the bat sign. It looks like a flying bat, shown in Figure 6 .

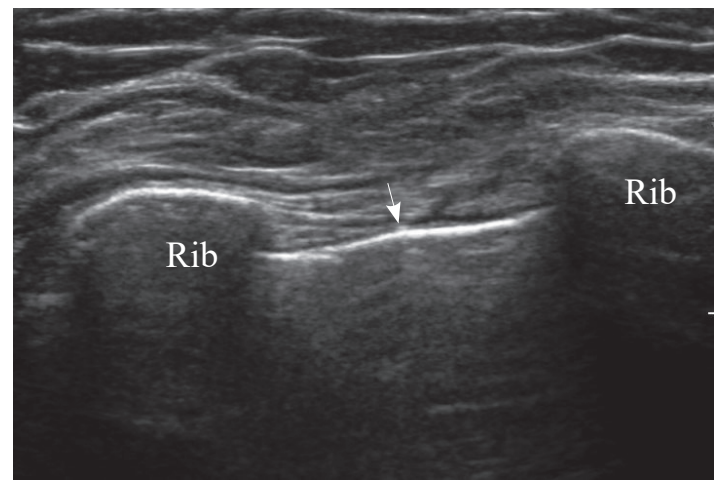

Figure 6 "Bat" sign of lung ultrasound. The grayscale sonogram shows two adjacent ribs and their shadows, and the pleural line on the surface of the lungs (arrow), all of which resemble bats with wings spread.

\subsubsection{Pulmonary sliding sign}

The hyperechoic normal lung-pleural line moves with the rhythm of breath, which is shown on the real-time ultrasound as a back and forth sliding line relative to the chest wall, which is called pulmonary sliding sign.

\subsection{5 "Seashore" sign}

It is caused by normal lung sliding and the relatively static chest wall. M-mode image usually shows a linear/ laminar pattern in the tissue superficial to the pleural line and a "sandy" appearance deep to the pleural line. This phenomenon is known as the "seashore sign" (Fig. 5B).

\subsection{Common abnormal lung sonograms \\ 3.4.1 Abolished pulmonary sliding}

When a pneumothorax occurs, the free air is out of the visceral pleura not controlled by respiratory activity.
Therefore, the normal lung sliding caused by breathing will be disappeared, and the hyperechoic interface will remain static. Besides pneumothorax, the disappearance of pulmonary sliding signs could also be seen in pleural adhesions, severe pulmonary fibrosis, apnea, and total atelectasis.

\subsubsection{Stratospheric sign or barcode sign}

It is typically found in patients with pneumothorax. Without the movement of free air, the lung sliding sign will be abolished, and the granular "sand beach" area formed by the sliding of the lungs disappeared and is replaced by densely distributed parallel line, termed Stratosphere of Barcode sign.

\subsubsection{Lung point}

When pneumothorax occurs, partially collapsed lungs can still inflate and deflate during respiratory cycle. When inhaling, the lung expands and pushes away the free air; when exhaling, the lung retracts and the free air occupies the position again. This separation point can be shown via ultrasound image dynamically, called lung point. The diagnosis of pneumothorax using lung point sign is moderately sensitive (66\%), but highly specific (100\%).

\subsubsection{B-lines}

Alveolar disease, pulmonary interstitial inflammation, and pulmonary interstitial fibrosis can alter the fluid-air composition of the interstitial and alveoli. When these changes involve visceral pleura, ringing artifact will be seen. It could be explained by a bubble-tetrahedron bugle mechanism. Specifically, when a small amount of fluid is trapped between four microbubbles, a "bulge" that is able to oscillate when struck by ultrasound will be formed. And ultrasound signals will constantly emit back to transducer, resulting in a strong vertical artifact. On sonogram, we can see hyperechoic points with posterior narrow-band-like strong echoes extending to the bottom of the screen without attenuation. They are called B-lines, and also called the vertical lines. B-lines can be seen in the intercostal space of the chest near the diaphragm on the normal lung but are not more than 2-3 lines.

\section{B3-Line and B7-Line}

When the distance between two adjacent B-lines is about $3 \mathrm{~mm}$, they are called B3-lines. If the distance is 6 to $7 \mathrm{~mm}$, they are called B7-lines. B3-lines represent early fluid accumulation at the alveolar level and little pulmonary function impairment, while B7-lines indicate an increase in fluid leakage to the lobular space with thickened septa and marked impairment in lung function. Sometimes the dense arrangement of the B-lines is difficult to distinguish and count. When confluent B-lines spread throughout the lung field, the sonogram shows a diffuse B-line pattern, called the white lung (Fig. 7).

\subsubsection{Shred sign}

The gas in the local alveoli is replaced by fluid 
(exudates, leakage, blood, etc.) to form a solid change. The solid part is irregular, and the boundary is ambiguous. Ultrasound image shows irregular hypoechoic area, like fragments.
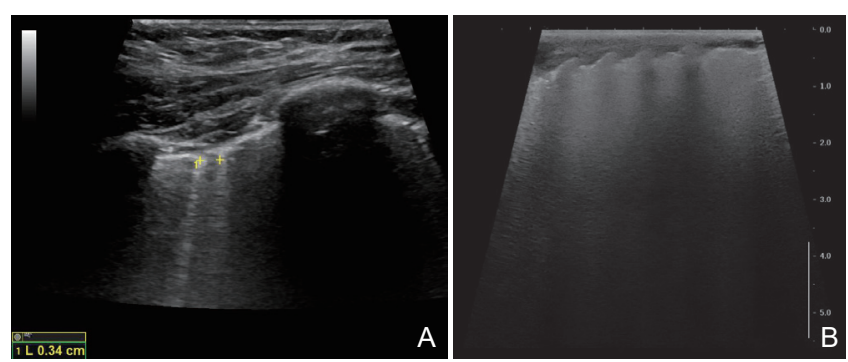

Figure 7 B-line and white lung. (A) Ultrasound shows a comet taillike strong echo originating from the pleural line and perpendicular to the pleural line. Here, the distance between the B-line is about 3mm (B3-line); (B) The B-line is densely distributed and cannot be distinguished from each other, and even the rib sound shadow is disturbed. The entire lung field is occupied by a "white" hyperechoic area (white lung).

\subsubsection{Solid tissue sign}

Collapsed or consolidated lung becomes non-aerated and echogenic, resulting in sonographic solid tissue change with clear boundaries and inner blood vessels, which is also called hepatization.

\subsubsection{Air bronchogram or fluid bronchogram}

These signs are caused by residual gas or liquid in the bronchi when lung consolidation occurs. If the residual gas in the bronchi moves with breathing, it is called dynamic air bronchograms. If fluid remains in the bronchi, it appears as low to non-echoing along the duct.

\subsubsection{Pleural effusion}

Different amounts of fluid accumulate in the pleural cavity. Ultrasound shows an echo-free space in the thoracic cavity with posterior echo enhancement. Due to different causes, floating echoic structures can be shown in the effusion. When a large amount of effusion is present, the compressed lung tissue can be found floating in the effusion.

Expert opinion: The content of the article is to analyze the ultrasound imaging characteristics of lungs under normal and pathological conditions in combination with the physical principles of ultrasound, referring to the literature on ultrasound imaging at home and abroad. (Recommended strength: B. Recommendation: Good evidence-based evidence can facilitate the analysis and judgment of clinical ultrasound images.)

\subsection{Lung ultrasound examination methods and steps}

Ultrasound scans of the lungs can be performed longitudinally and laterally. Longitudinal scan requires the probe to be placed perpendicular to both chest wall and the intercostal space, while lateral scan requires the probe to be placed perpendicular to the intercostal space and parallel with the intercostal space. Commonly used ultrasound examination methods and procedures are in accordance with the BLUE-PLUS scheme, including a unilateral 6-zone scheme and an 8-zone scheme.

\subsubsection{BLUE-PLUS scheme}

French scholar Lichtenstein summarized the examination protocol for bedside lung ultrasound in emergency (BLUE). Methods and procedures: the position of the examiner's hand and chest wall are used to visually set the BLUE and posterolateral alveolar/pleural syndrome (PLAPS) points. Compared to $\mathrm{CT}$, this protocol can find most of the pulmonary lesions and pleural lesions [9,10]. Disadvantages: The ultrasound checkpoint is limited by the size and shape of the examiner's hand and the position of the patient's diaphragm.

\subsubsection{Unilateral six-zone scheme and prone position unilateral eight-zone scheme}

The unilateral lung is divided by the front axillary line and the posterior axillary line into three regions, the anterior lung, the lateral lung, and the posterior lung. It is further divided into upper and lower parts, that is, a total of six areas: anterior up, anterior down, lateral up, lateral down, posterior up, and posterior down [11] (Fig. 8). Since COVID-19 patients' lung lesions are usually bilateral, peripheral, and multifocal, unilateral eightzone method are commonly used for lung ultrasound examination and lung recruitment assessment. For critically ill patients, unilateral six-zone scheme is more widely used.
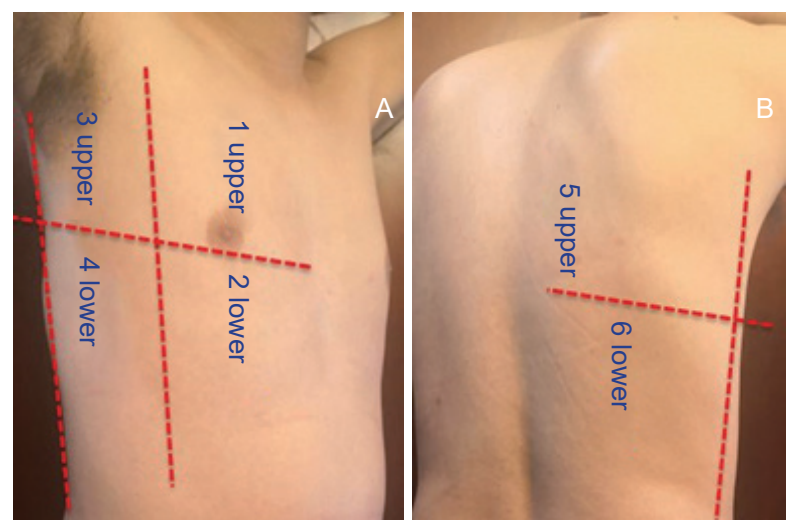

Figure 8 Partitioning methods for unilateral six-zone scheme. (A) supine position; (B) lateral position. Lung regions are divided by anterior and posterior axillary lines into anterior lung, lateral lung, and posterior lung. Three regions were further divided horizontally into 6 parts.

Expert opinion: The content of the clause refers to the methods and steps of the ultrasound examination of normal lungs and lungs with severe disease at home and abroad, and analyzes several methods and steps of lung ultrasound examination. (Recommended strength: B. Recommendation: Good evidence-based evidence can facilitate clinical ultrasound image acquisition and avoid missed diagnosis.) 


\subsection{Ultrasound features of COVID-19}

COVID-19 ultrasound features are similar to other viral pneumonia and bronchopneumonia. The ultrasound findings represent the changes of air-fluid ratio in the lung, and it varies with the natural course of the disease.
The main characteristics are shown in Figure 9.

\subsubsection{Pleural line and A-line}

Thickened pleura, rough echo, interruption of normal smooth pleura, and disappearance of A-lines and pulmonary sliding signs.
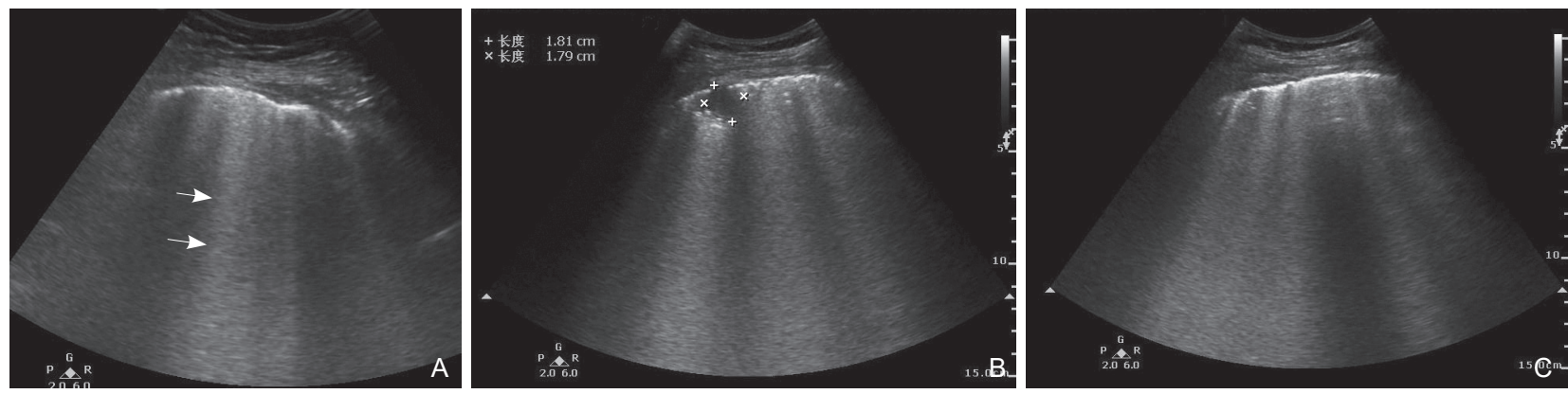

Figure 9 COVID-19 ultrasound images. (A) Thickened and rough pleural line, A-line disappeared with and increased number of B-lines (arrows); (B) Consolidation of the subpleural lung (indicated by measurement cursors); (C) Thickened pleural lines, interrupted echo, and increased and merged B-lines with the interval $\leqslant 3 \mathrm{~mm}$.

\subsubsection{B-line}

Increased number of B-line, appearance of B7-lines (indicating potential interstitial pulmonary edema), denser B3-lines (indicting potential alveolar pulmonary edema), and white lung formed by B-line fusion.

\subsubsection{Pulmonary consolidation}

Subpleural atelectasis and B-lines, pulmonary consolidation with shred signs and air bronchogram, and liver-like changes (hepatization).

\subsubsection{Pleural effusion}

Often small or limited pleural effusion.

\subsection{Ultrasound assessment of COVID-19 severity}

COVID-19 lung lesions change along with the severity of the disease, and multiple ultrasound signs can develop at the same time or interchangeably. Ultrasound is an important clinical imaging modality which can evaluate the real-time progress of the disease at the bedside and adjust the treatment plan in time.

\subsubsection{Early stage of COVID-19 (0-4th day)}

Clinically, patients may develop dry cough, chest tightness, and low-grade fever [12]. Ultrasound findings typically include irregular, blurry, or interrupted pleural lines, subpleural small patchy hypoechoic shadows with unclear borders, and subpleural multiple or fused B-lines (showing a waterfall sign) [13]. The lesions are usually fixed with one or more lung regions involved (Fig. 10).

\subsubsection{Early advanced stage of COVID-19 (5th-8th} day)

The symptoms of this stage include cough, shortness of breath, and fever [12]. Ultrasound shows peripheral pleural line interruption, subpleural multiple continuous patch-like, nodular, and bar-like irregular hypoechoic changes, air bronchogram sign, multiple or fused B-lines (waterfall sign) in fixed positions [13]. Single or multiple lung regions are involved with bilateral lower lung fields the most common. Limited pleural effusion and thickened pleura can be seen in the pleural cavity adjacent to some lesions (Fig. 11) [14]. Color Doppler flow imaging (CDFI) shows blood flow signals in the consolidation areas are more scarce compared to lobar pneumonia lesions.
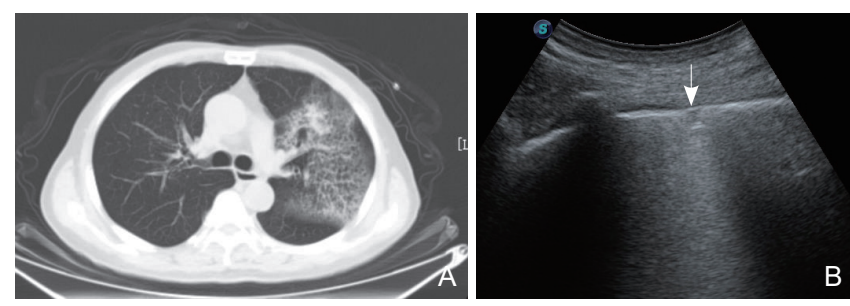

Figure 10 Early stage COVID-19 ultrasound images. (A) An 81-year-old male COVID-19 patient developed fever up to $37.8^{\circ} \mathrm{C}$. His high-resolution CT (HRCT) showed sub-pleural ground glass-like mass in the left upper lung, with reticular appearance; (B) Ultrasound using a convex array probe showed the left posterior lower lung area has a large lung circumferential B-line, A-line disappears, small patchy lesions around the lung, and pleural line is not continuous (shown by arrow).

\subsubsection{Advanced stage of COVID-19 (10th-13th day)}

Clinical symptoms get worsened with dyspnea and fever [12]. Ultrasound findings include peripheral interrupted or disappeared pleural line, expanded subpleural consolidations, multiple continuous largescale hypoechoic consolidation with hepatization sign [15], large band-like and small patchy shadows with diffused B-lines (white lung), and extensive air bronchogram sign. In the advanced stage, multiple lung areas are involved, and bilateral lower lung fields become denser, indicating worsening consolidation. In addition, free pleural effusion and thickened pleura could be seen in some patients (Fig. 12); CDFI shows dendritic 
blood flow signals near the hilar area, but not inside the pulmonary consolidation lesions.

Expert opinion: The content of the article introduces the ultrasound characteristics of COVID-19 and the ultrasound manifestations at different stages of progression, consistent with CT findings. (Recommended strength: A. Strong recommendation: Positive evidence-based evidence is conducive to improving the recognition, analysis and judgment of COVID-19 pneumonia ultrasound images.)
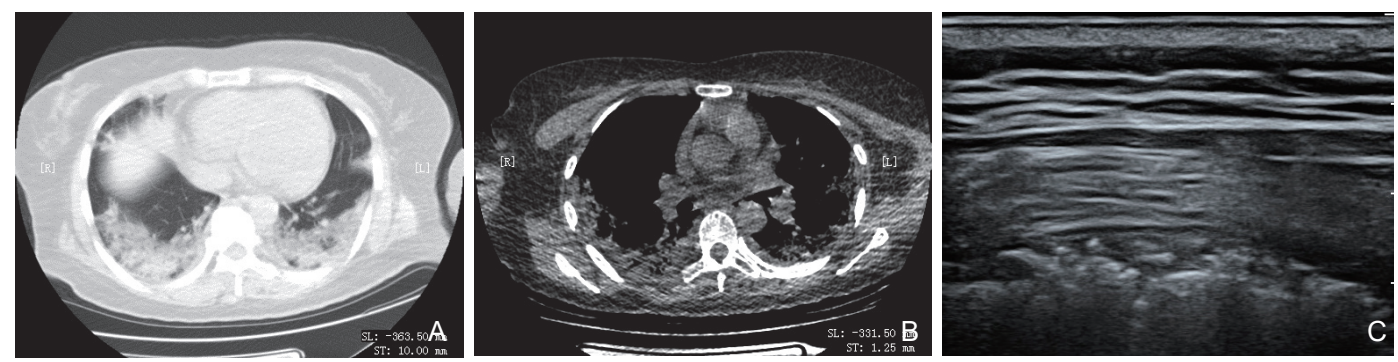

Figure 11 Early advanced stage of COVID-19 ultrasound images. (A and B) CT images of lung window and mediastinal window. A 42-year-old female COVID-19 patient on the 7th days of fever with body temperature up to $38.5^{\circ} \mathrm{C}$. High-resolution CT (HRCT) showed right upper lobe subpleural heterogenous band-like shadows; (C) Ultrasound using a linear array probe showed interrupted and disappeared pleural line in the upper right lung field and a subpleural strip-like faint solid lung tissue. Air bronchogram sign and B-lines were also seen.
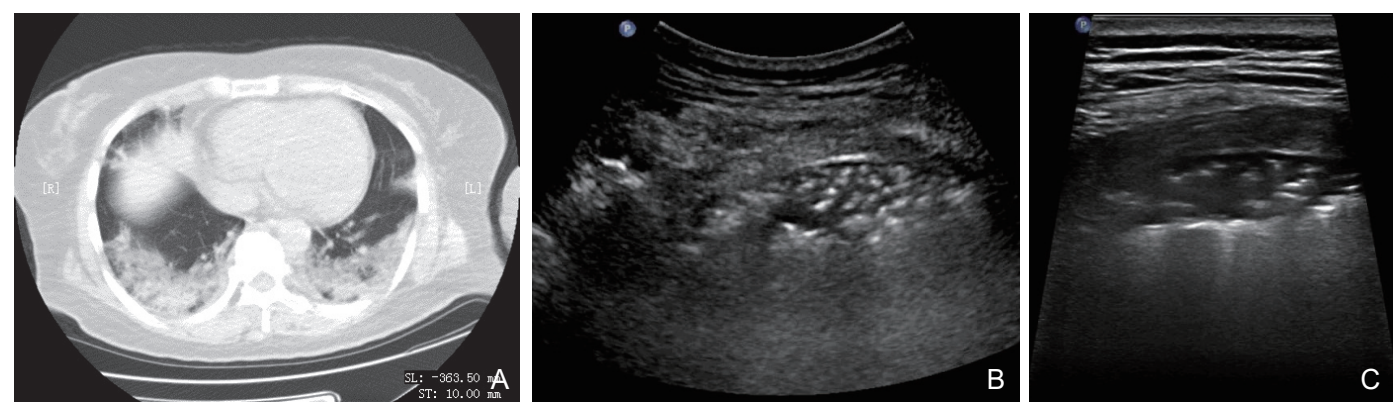

Figure 12 COVID-19 critical stage imaging performance. (A) 42-year-old female COVID-19 patient on the 13th day of fever with body temperature up to $38.5^{\circ} \mathrm{C}$. CT showed widespread lung lesions, bilateral large laminar hypoechoic consolidation in posterior lung fields, and air bronchogram sign; (B and C) Ultrasound showed hypoechoic consolidation area with posterior fused B-line.

\subsection{Myocardial injury and ultrasound characteristics of COVID-19}

Clinicians should be aware of the potential rapidly deterioration in some patients, which usually happens about a week after the disease onset. About $31 \%$ of critically ill patients develop cardiac injury [16]. Thus, bedside echocardiogram plays a crucial role in providing easily obtainable and clinically relevant information about hemodynamics (cardiac, lung, and vascular function) in order to real-time monitor and effectively treat critically ill COVID-19 patients.

3.8.1 Essentials of ultrasound assessment in critical ill patients with COVID-19

1) Focused echocardiography assessment on left ventricular function and right ventricular function: a. Visual inspection of the left ventricular global systolic function is recommended for patients with normal wall motion; $b$. The M-mode ultrasound to assess left ventricular function is recommended for patients with diffuse wall motion abnormalities; c. The Simpson single or biplane method to assess left ventricular function is recommended for patients with segmental wall motion abnormalities. d. If necessary, visual measure of the right ventricular wall motion or M-mode method to measure the tricuspid annular plane systolic excursion (TAPSE) of the tricuspid annulus and estimate the right ventricle fraction of area change (FAC) by the two-dimensional echocardiography should be performed.

2) Assessment of pulmonary artery systolic pressure (PASP): on the condition of no right ventricle outflow stenosis, using tricuspid valve regurgitation pressure to measure the systolic pressure of pulmonary artery.

3) Assessment of the inferior vena cava diameter and its respiration collapsibility.

4) Quickly identify the presence of pericardial effusion and its localization on the subcostal and parasternal views.

5) Assess cardiac structure abnormalities and determine whether it is accompanied with acute or chronic heart disease. A comprehensive echocardiogram measurement is not recommended. 


\subsubsection{Echocardiography characteristics of cardiac} injury in patients with COVID-19

1) Left ventricular dysfunction: The enlarged left heart chamber, the abnormal ventricular wall motion, and the reduced systolic function are shown in echocardiogram (Fig. 13). People those have a history of cardiovascular disease or new-onset cardiovascular injury can develop myocardial infarction with mural thrombosis formation (Fig. 14A).

2) Right ventricular dysfunction: The enlarged right heart chamber, PASE, and the right ventricular systolic dysfunction (Fig. 14B).
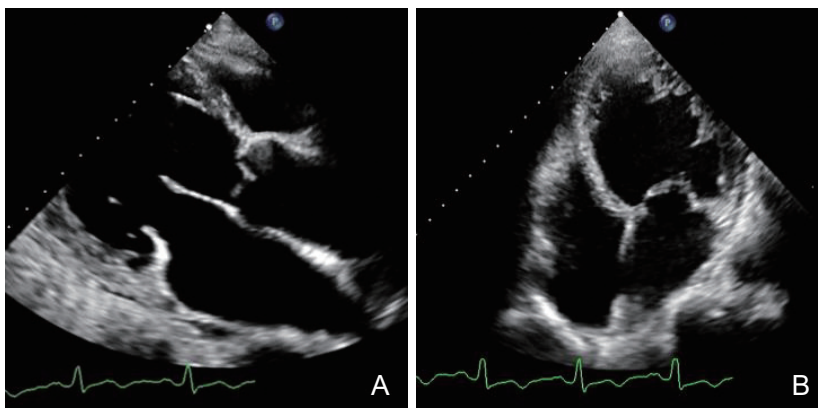

Figure 13 COVID-19 with cardiac abnormalities. A 73-year-old male COVID-19 patient (positive nucleic acid test, CRP: $170 \mathrm{mg} / \mathrm{L}, \mathrm{BNP}: 1077$ $\mathrm{pg} / \mathrm{mL}$ ); (A and B) significantly enlarged left heart on the parasternal longaxis view and apical four-chamber view.
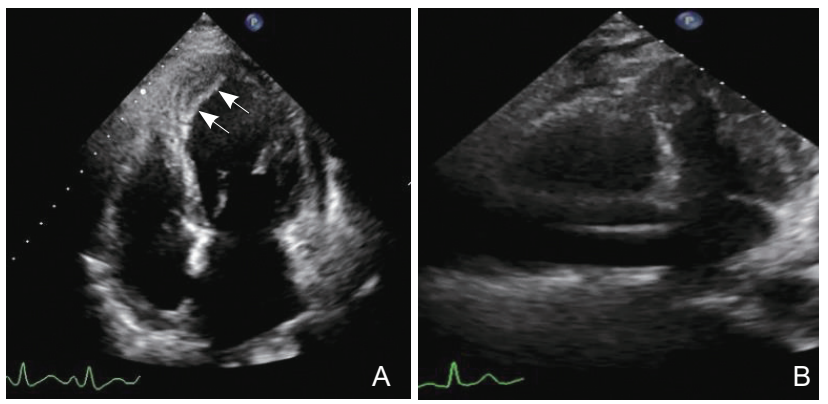

Figure 14 COVID-19 with cardiac abnormalities. (A) A 45-year-old female COVID-19 patient (positive nucleic acid test, D-dimer: $3.39 \mu \mathrm{g} /$ mL,TNI: $9889.4 \mathrm{ng} / \mathrm{L}$, BNP: 632 pg / mL). Left ventricular thrombosis formation on the apical four-chamber view (indicated by arrows); (B) A 69-year-old male COVID-19 patient (positive nucleic acid test, respiratory failure, D-dimer: $6.82 \mu \mathrm{g} / \mathrm{mL}, \mathrm{BNP}: 632 \mathrm{pg} / \mathrm{mL}$ ). Echocardiogram showed significantly enlarged right heart on the apical four-chamber right ventriclefocused view.

3) Pulmonary hypertension: Ultrasound findings include enlarged main branch of pulmonary artery and right ventricle, increased tricuspid valve regurgitation pressure, and interventricular septum deviated to the right (D-shaped left ventricle) (Fig. 15).

4) Pericardial effusion and pericardial tamponade: The typical features include swinging heart, diastolic collapse of the right atrial and ventricular wall, inspiratory septal bounce (interventricular septum moving toward the left ventricle during inspiration and toward the right ventricle during expiration), and the dilated inferior vena cava.

5) The abnormalities indicating arrhythmia or other underlying diseases and its complications.
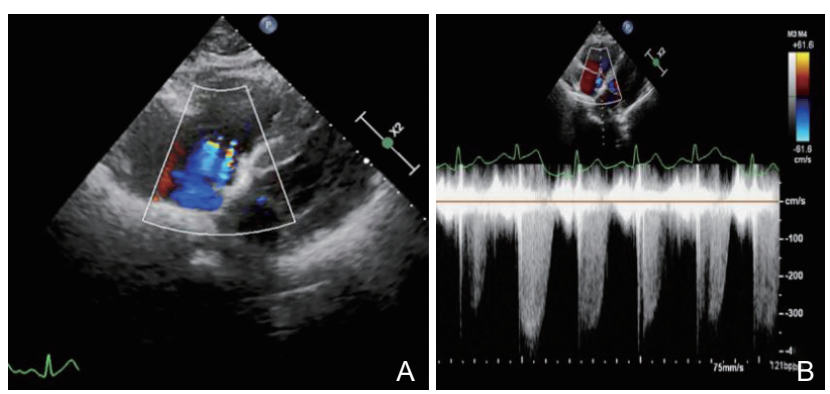

Figure 15 COVID-19 with pulmonary hypertension. The same patient as in Figure 14B, (A) Color Doppler echocardiography showing moderate tricuspid regurgitation; (B) The increased pulmonary arterial systolic blood pressure was estimated by tricuspid regurgitation pressure gradient.

Expert recommendations: The content of the clause is based on the acquisition of bedside echocardiography images of patients with COVID-19 in the Department of Ultrasound, Union Hospital of Tongji Medical College, Huazhong University of Science and Technology, Wuhan, China. (Recommended strength: A. strongly recommended: positive evidence-based can improve clinical outcomes, and the advantages outweigh the disadvantages.)

\subsection{Pediatric lung ultrasonography}

At present, infants and children are also considered to be susceptible to COVID-19 $[17,18]$. The application of pulmonary ultrasound in pediatrics is relatively mature. Ultrasound can also identify other common pediatric pulmonary diseases, such as neonatal respiratory distress syndrome (NRDS), meconium aspiration syndrome, transient tachypnea of the newborn, and neonatal pneumothorax.

The characteristics of normal lung ultrasound images are the same as those of adult lung ultrasound, see above. It should be noted that sparse B-lines can appear in some lung fields within 3-7 days of birth of newborn babies, and disappear after a few days with development (Fig. 16).

The common features in children's lung ultrasonography include: 1) Pleural line thickening, blurring, continuously discontinuity sign in the lung field, and the disappearance of A-lines may be detected. 2) B-lines of varying densities appear in the lung field, ranging from sparse to fused B-line, alveolar-interstitial syndrome, and even "white lung". 3) Lung consolidation, with or without bronchial gas phase and bronchial liquid phase. It can be confined to the subpleural space, showing low-echo areas with irregular shapes and different sizes with patchy, snowflake-like gas strong echoes, or "shred 


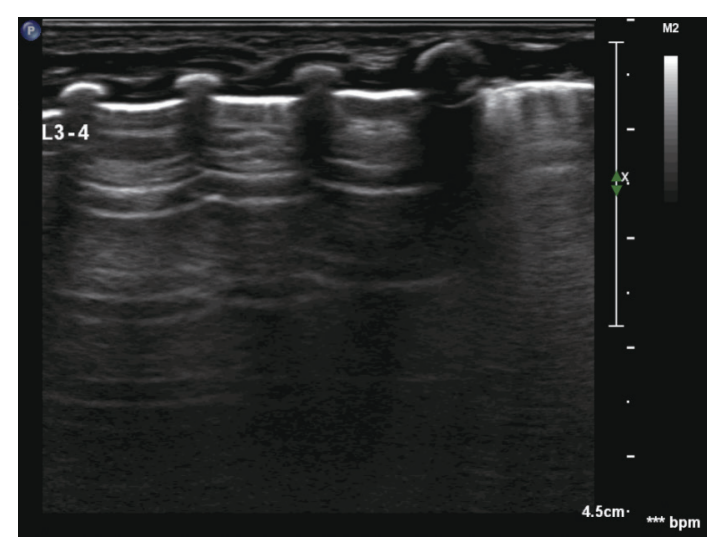

Figure 16 Ultrasound examination of newborn lung. Sparse B-lines could be seen in the local lung field by ultrasound (arrow).
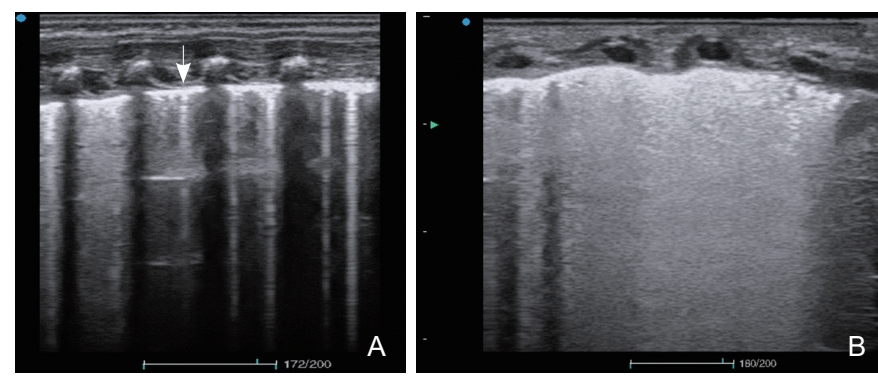

signs". Larger consolidations can appear lumpy. 4) Pleural effusions of different degrees can occur on one or both sides. 5) During the recovery period, the area of original consolidation may shrink, and the B-line density may gradually decrease and disappear. In addition, the "double lung-point" sign may appear in the junction of A-lines and B-lines, indicating the dividing point between normal and edema areas (Fig. 17).

Expert opinion: The article introduces the ultrasound manifestations and differential diagnosis of pediatric pneumonia. (Recommended strength: B. Recommendation: Positive evidence-based imaging could be helpful in analysis and judgment of clinical ultrasound images.)
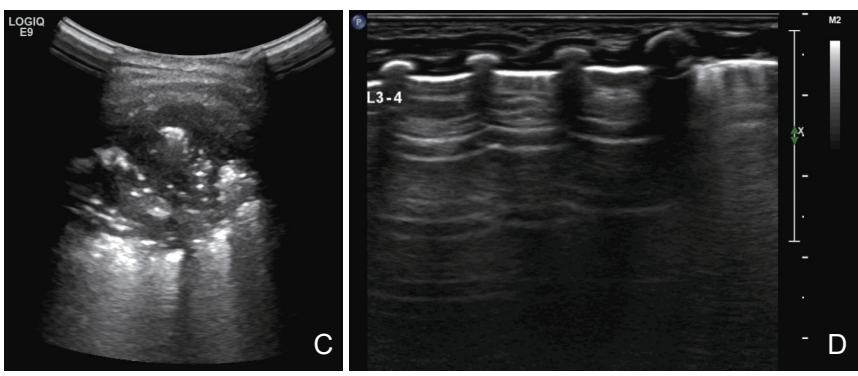

Figure 17 Ultrasound examinations of the lungs of the newborn. (A) The pleural line of the lung field was thickened and blurred (indicated by the arrow), and the A-line disappeared; (B) B-lines of different densities represented different degrees of alveolar-interstitial syndrome changes, and even "white lung"like changes; C: Consolidation of the lungs to the deep part of the lung field, showing a mass-like lesion; D: Double-lung-points were shown by ultrasound, indicating the boundary between normal lung tissue and tissue with increased water content.

\subsection{Ultrasound Blood Volume Assessment}

Volume assessment includes the assessment of volume status and fluid responsiveness. The volume status is a static maker, referring to the preload status related to the end-diastolic left ventricle volume. Common evaluation indicators include left ventricular end-diastolic diameter (LVEDD) and volume (LVEDV), and the diameter of the inferior vena cava (DIVC). In patients with hypovolemia, the DIVC becomes narrower. In the state of spontaneous breathing, DIVC less than 9 $\mathrm{mm}$ indicates low blood volume. LVEDD $<35 \mathrm{~mm}$, and the appearance of papillary muscle kiss signs, etc., may also indicate volume insufficiency.

Fluid responsiveness is a dynamic maker, referring to the ability of the left ventricle to increase its stroke volume (SV) in response to fluid infusion. It is a comprehensive reflection of preload status and cardiac function. After increasing circulating volume, the increase of cardiac output (CO) or stroke volume (SV) greater than $10-15 \%$ indicates good fluid responsiveness. Other common evaluation indexes of ultrasound include inferior vena cava diameter with respiratory variability, time-integrated respiratory variability $(\Delta \mathrm{VTI})$, and SV variability (SVV) of left ventricular outflow tract velocity $[19,20]$. Inferior vena cava variability is often reflected by the collapse index (cIVC) and dilation index $($ dIVC $)[21] . \operatorname{cIVC}=($ Dmax-Dmin $) /$ Dmax. $\operatorname{cIVC}>$ $40 \%$ in the state of spontaneous breathing indicates the patient may benefit from rehydration therapy. In the case of mechanical ventilation without spontaneous breathing, the respiratory variability formula of the inferior vena cava is $\mathrm{dIVC}=(\mathrm{Dmax}-\mathrm{Dmin}) / \mathrm{Dmin}$, with a cut-off value of $18 \%$. Based on heart-lung interactions, apnea test, a passive leg raise test, or a fluid challenge, SVV and left ventricular outflow tract $\Delta$ VTI estimation are also important indicators for assessing volume responsiveness, with a cut-off value of $12 \%$ to $15 \%$.

\section{$\triangle \mathrm{VTI}$ is calculated as:}

$$
\Delta V T I=\frac{\left(2 \times\left(V T I_{\max }-V T I_{\min }\right)\right.}{\left(V T I_{\max }+V T I_{\min }\right)} \times 100 \%
$$

It should be noted that ultrasound indicators can be used for volume status or fluid responsiveness assessment, but they cannot completely replace other assessment methods, such as blood pressure, central venous pressure, pulmonary artery wedge pressure, and continuous cardiac output monitoring (PiCCO). Thus, comprehensive analysis should be carried out in combination with each other. 


\subsection{Interventional ultrasonography}

\subsubsection{Serous membrane effusion}

COVID-19 causes fewer cases of serous fluid (including pleural, peritoneal and pericardial effusions), but multiple organ failure and multiple serous fluid may also be present in severe patients. Ultrasound can confirm the diagnosis and perform interventional ultrasound treatment according to clinical needs.

1) Indications: Those who need biopsy, fluid drainage, catheter placement, thermal ablation, paracentesis, and medication injection, et al.

2) Contraindications: Those with severe coagulopathy, very little effusion $(<1.0 \mathrm{~cm})$, or without a puncture path.

3) Position: Patients with pleural effusion should be placed in a sitting position. Those with wrapped pleural effusion can be placed in any position; those with abdominal peritoneal effusion and pericardial effusion can be placed in semi-recumbent, supine, or lateral position.

4) Operation steps: Before using the ultrasound probe, conventional disinfection, laying of sterile towels, and 1\% to $2 \%$ lidocaine local anesthesia should be performed. The ultrasound probe was applied with coupling agent and placed in a disposable sterile cover. For ascites, percutaneous transhepatic cholangiography (PTC) needle is guided by ultrasound and percutaneously punctured into the effusion. The needle position was monitored in real time, and the fluid was drawn up using a syringe. The needle tip was gradually retracted when the effusion completely disappeared. Those who need a tube for drainage, after successful puncture, a tube can be placed via Seldinger method (Fig. 18 and Fig. 19). The specific steps are as follows: see the effusion-pull out the needle core - insert the guidewire - pull out the needle sheathexpand the needle channel - place the drainage tube along the guidewire - exit the guidewire-connect the drainage bag [22].

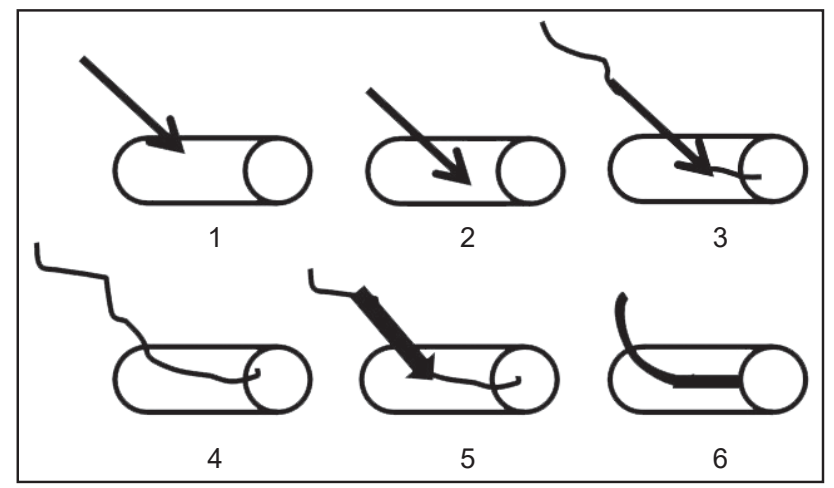

Figure 18 Seldinger method.
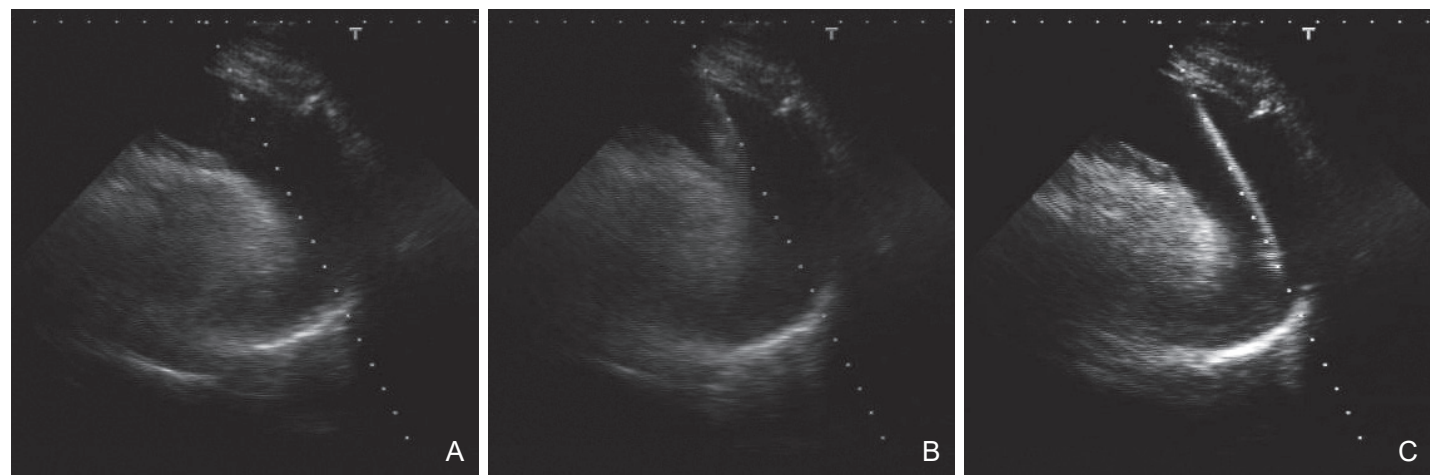

Figure 19 Ultrasound-guided pericardial effusion puncture catheter. (A) Determine the location of the effusion; (B) Insert the puncture needle; C: Insert the drainage tube.

5) Common complications: By real-time ultrasound guidance, the complications will be very rare, including infection, bleeding, and damage to surrounding organs.

\subsubsection{Ultrasound-guided peripheral and central} venous catheter placement

In emergency and critical care settings, ultrasoundguided peripheral and central venous catheterization can quickly establish venous channels [23,24].

1) Indications: When blind venous penetration is difficult but blood products or intravenous infusions are urgently needed. Also for those who need long-term central venous catheterization and thrombolysis.

2) Contraindications: Patients who have multiple thrombophlebitis, or the venous access is injured. Others are same as " fluid drainage" (shown above).

3) Operation steps: The basic procedure is the same as that of "serous membrane effusion drainage". Do not repeat puncture at the same puncture point to avoid accidental injury to the artery and furthering hematoma and bleeding. After placing the tube, the adjustment clamp must be closed to prevent air from entering and the forming of an air plug [25].

4) Complications: By real-time ultrasound guidance, the complications will be very rare, including infection, bleeding, and damage to surrounding organs.

3.11.3 Application of ultrasound in extracorporeal membrane oxygenation (ECMO)

The core ECMO circuit needs vascular access, 
connecting tubing, a blood pump, and a gas exchange device which is similar to the heart-lung by-pass machine used in open-heart surgery. The blood that passes through the gas exchange is pumped back into the vein (venovenous, VV mode) or artery (veno-arterial, VA mode) [26]. When using the VA mode, the femoral artery and vein are mostly punctured, while the femoral vein and the jugular vein are mostly chosen in VV mode [27].

3.11.4 The steps of ultrasound-guided puncture and catheterization of arteries and veins

Likewise, Seldinger method is utilized (Fig. 20). In the VA mode, in order to avoid ischemia of the ipsilateral distal limb caused by the built-in tube of the femoral artery, a distal arterial perfusion tube is often required. At this point, because there is a catheter in the femoral artery, the distal femoral artery diameter is thin and the blood flow is decreased. Ultrasound-guided approach is an accurate and effective way to safely initiate the puncture of the blood vessels and evaluate the catheter tip placement in real time. The tip of the catheter should pass through the inferior vena cava, and reach the entrance of the right atrium.
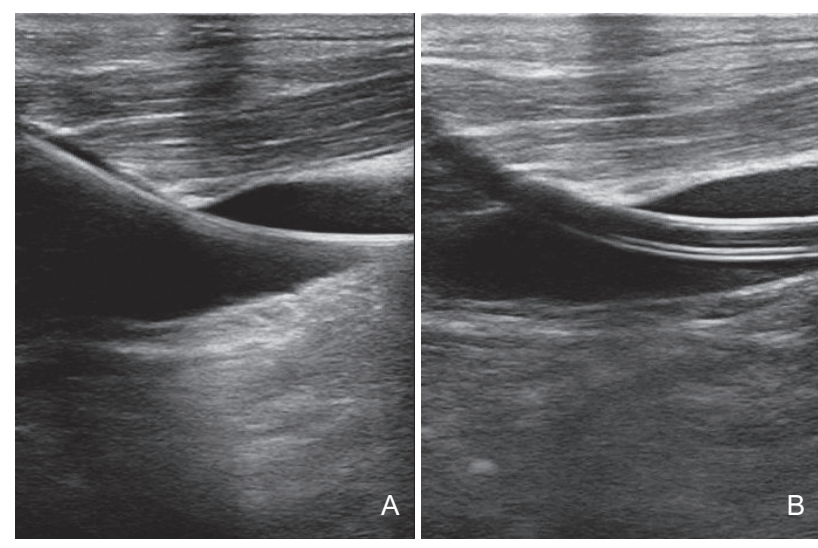

Figure 20 Ultrasound-guided internal jugular vein puncture and placement. (A) Place the guidewire along the puncture needle under ultrasound guidance; (B) Place the catheter along the guidewire under ultrasound guidance.

After the initiation of ECMO, ultrasound can be used to timely monitor cardiac function and lung volume and to evaluate the pleural effusion before weaning patients off ECMO. IJsselstijn et al. had explained the detailed information about improving long-term outcomes ECMO [28].

Expert opinion: The content of the clause refers to the application methods and steps of interventional ultrasound worldwide, and it combines a large number of clinical work practices. (Recommended strength: A. Strong Recommendation: Positive evidence-based evidence can improve clinical outcomes, and the advantages outweigh the disadvantages.)

\section{Ultrasound Staff Protection}

In the course of diagnosis and treatment, because ultrasound practitioners often work closely with patients, coupled with the increasing number of patients and relatively poor ventilation in the working area, the risk of occupational exposure is undoubtedly high.

\subsection{Diagnostic ultrasound examination area}

The Diagnostic ultrasound working area can be divided into staff-only access, clean area, buffer area, examination area, and a buffer hallway linked between examination area and buffer area. All ultrasound providers should enter the clean area via staff-only access and leave their personal belongings in the clean area. In the buffer area, medical staffs need to put on personal protective equipment (PPE) outside their proper working clothes before entering the examination area. After ultrasound examination, the staffs will enter the buffer area through the buffer hallway to take off their PPE and working clothes, and then go back to the clean area. Hand hygiene should be performed before and after all patient contact, contact with potentially infectious material, and before and after removing PPE.

\subsection{Triage and waiting area}

1) Self-protection for triage receptionists and nurses: Secondary protection, keeping the environment ventilated, and timely hand hygiene.

2) Triage area management: All patients answer the serial travelling history and symptoms related questions and have their body temperature checked before entering the waiting area. If a patient's body temperature is above $37.3^{\circ} \mathrm{C}$ or having any suspicious travelling history/ symptoms, he/she should be guided to the fever clinic for COVID-19 screening.

3) Waiting area management: Remind patients and their attendants to sit separately and wear masks; People from inpatients and outpatients should be arranged to stay in different areas to wait for examination.

\subsection{Consultation room}

1) Medical personnel should wear working clothes, working caps (operating caps), medical surgical masks during general examinations. If necessary, isolation gown and goggles, latex gloves should be put on. Don't forget perform hand hygiene.

2) The surface and floor of the consultation room must be wiped with chlorine-containing disinfectant twice a day. Consultation rooms can be ventilated with air purification sterilizers 2 or 3 times a day for at least 30 minutes per time and disinfected with ultraviolet disinfection lamps.

3) Make sure the disposable examination sheet is replaced before next patient's examination. The ultrasound probes need to be disinfected after each 
examination. All the medical wastes in the consultation room should be classified and put into special bags in accordance with the "Measures for the Management of Medical Waste".

4) Fevered patients who have been excluded from infection of COVID-19 need to be arranged for a separate room.

\subsection{Isolation ward}

1) Comply with the protection requirements for medical personnel in the isolation area once personals step into isolation wards. Working in the area of isolation needs level 3 protection, which mainly includes wearing disposable working caps, goggles (anti-fog type) or protective face screens, medical protective masks (N95), disposable protective suits, disposable latex gloves, disposable boot covers and shoe covers. If there is high risk of body fluid/secretion or blood spill, a comprehensive respirator guard should be used.

2) Put on and take off the PPE process (Fig. 21).

\begin{tabular}{|c|c|c|c|}
\hline \multicolumn{2}{|c|}{ The process of putting on protective suit } & \multicolumn{2}{|c|}{ The process of taking off protective suit } \\
\hline \multirow{5}{*}{ First buffer } & Wear a cap & \multirow{5}{*}{ Third buffer } & Wash hands and take off outer latex gloves \\
\hline & Wear a mask (N95/N99) & & Wash hands and take off protective goggles or face screen \\
\hline & Change overalls & & Wash hands and take off boots covers \\
\hline & Wear inner latex gloves & & Wash hands and take off protective suit \\
\hline & Change shoes & & Wash hands \\
\hline \multirow{5}{*}{ Second buffer } & Wear protective suit & \multirow{5}{*}{ Fourth buffer } & Wash hands and change shoes \\
\hline & Wear protective goggles or face screen & & Wash hands and take off inner latex gloves \\
\hline & Wear outer latex gloves & & Wash hands and take off overalls \\
\hline & Wear boot covers & & Wash hands and take off mask \\
\hline & & & Take off cap and wash hands \\
\hline
\end{tabular}

Figure 21 The procedure for putting on and removing personal protective equipment.

3) The removed PPE should be placed in a designated disposable collection bag for centralized disposal, referring to the Regulations on the Administration of Medical Wastes and Measures for Medical Wastes Management of Medical and Health Institutions.

\section{Cleaning and Disinfection of Ultrasound Equipment}

Wear gloves and goggles when disinfecting and avoid touching the skin and eyes.

\subsection{Control panel and monitor of ultrasound instrument}

After turning off the ultrasound machine, either using a disinfectant wipe or spraying the disinfectant onto the disinfection area and wipe it off with a soft tissue. Of note, do not spray the too much disinfectant into the machine from the joints of the control panel.

\subsection{Ultrasound probe}

It is recommended to use a disposable probe covering during each ultrasound examination. After examination, remove the cover and wipe the probe with paper towel to avoid cross-infection. The surfaces of the probes should be disinfected by the disinfectant containing quaternary ammonium salts or hydrogen peroxide. Recommended disinfectant includes: a. 70\% isopropyl alcohol (IPA) or 75\% alcohol for disinfection of mainframe and cable of ultrasound machine. b. glutaraldehyde (2.0-2.5\%) or phthalaldehyde $(0.55 \%)$ for disinfection of ultrasound probe.

Expert opinion: The protection of the ultrasound physician shall comply with the protection requirements of the medical personnel in the isolated area. The ultrasound department in the epidemic period needs to do well in the management of the diagnosis area and personnel protection. (Recommended level: A. Strong recommended. Ultrasound medical staff should be strongly recommended that start from the details of their own protection to prevent problems before they occur.)

\section{Remote Ultrasound for Management of the COVID-19 Patients}

Remote delivery of ultrasound services is a growing area of interest. With the development of information technology, Internet, artificial intelligence (AI), cloud computing, $5 \mathrm{G}$, and other emerging technologies, transferring images to a long-distance server or the cloud for data analysis and remote consultation is no longer a dream. There is no doubt than ultrasound providers could benefit from the real-time sharing of ultrasound data and mutual transmission and interconnection of ultrasound image data [29]. 


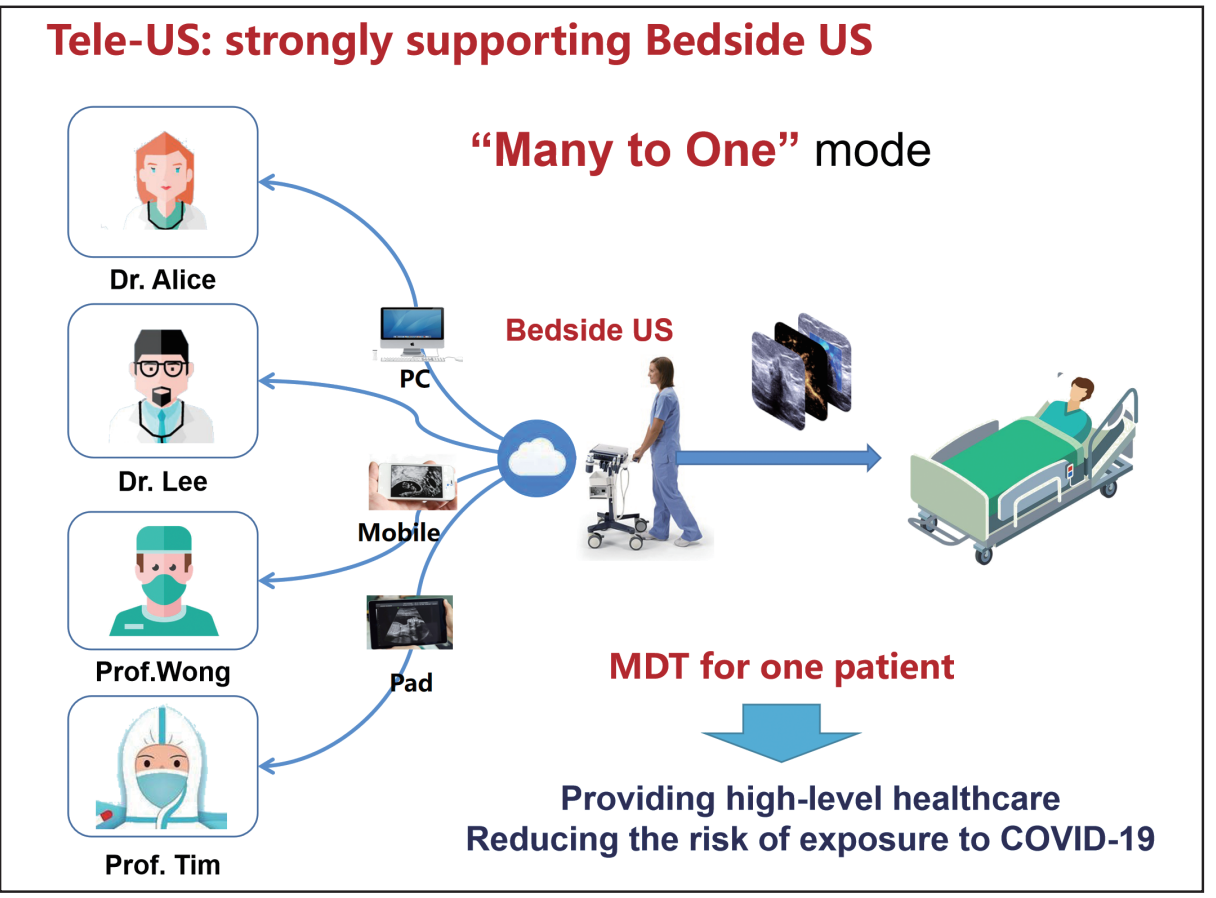

Figure 22 Schematic diagram of the basic structure of remote ultrasound.

\subsection{Basic architecture}

The diagram of the basic architecture and data management mode of remote ultrasound is shown in Figure 22. Through the remote ultrasound cloud platform, digital imaging and communications in medicine (DICOM) data of ultrasound images can be instantly stored or retrieved, uploaded or downloaded, and analyzed or archived.
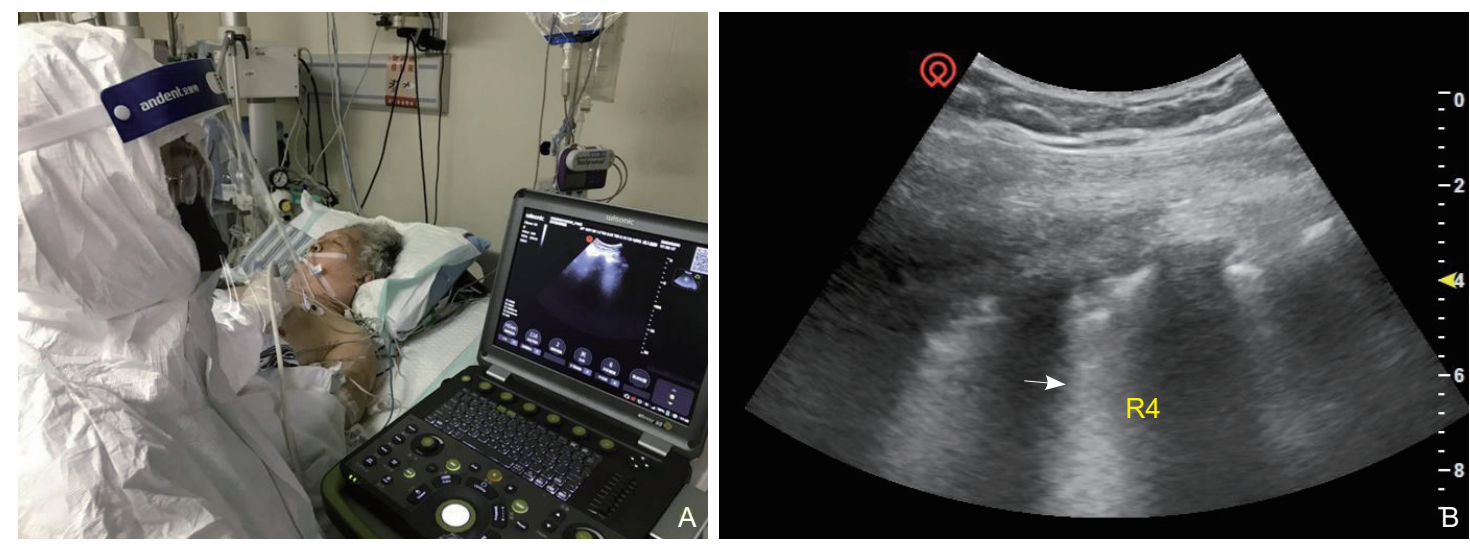

Figure 23 Remote ultrasound consultations for COVID-19 patients. (A) A 67-year-old female, diagnosed with COVID-19. The sonographer at the patient's end performed a pulmonary ultrasound examination, and the radiologists stayed at the end of the consultation giving real-time expert opinion; (B) The BLUE point on the left anterior chest showed a significant increase in the B-lines and the fusion (white arrow), and localized pleural thickening with irregular borders.

\subsection{Application of remote ultrasound in COVID-19}

In the course of treating the patients with COVID-19, remote ultrasound can help to decrease ultrasound providers' risk to potential exposures. The combined use of remote ultrasound and tele-operated robotic ultrasound can minimize the cross-infection between ultrasound practitioner and ward patients and among healthcare workers. It could also be easily used for real-time monitoring of the disease progress, such as pulmonary and cardiac function, volume assessment, and evaluation and follow up after interventional operations, etc. (Figure 23 and Figure 24).

The shortcomings of remote ultrasound: 1) Remote ultrasound largely dependent on the technique of the sonographer at the patient end and the level of proficiency of radiologist operating the robotic arm remotely; 2) Remote ultrasound also needs high resolution images and fast Internet connection; 3) Data 

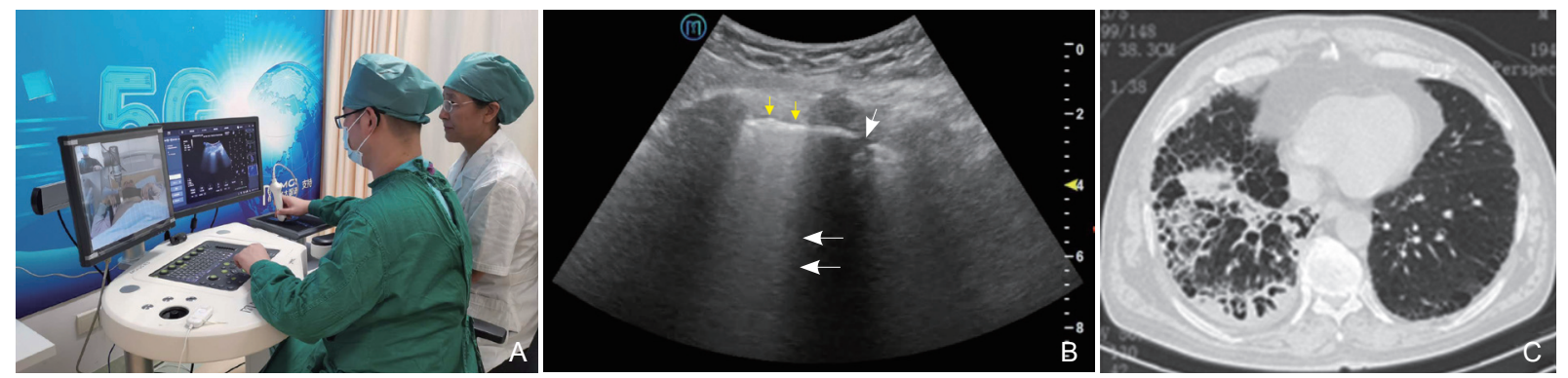

Figure $245 \mathrm{G}$ remote robotic ultrasound of a suspected COVID-19 patient. (A) A 75-year-old male patient, suspected of having COVID-19. The radiologist was on the doctor's side operating the robotic arm to perform the remote ultrasound exam. The distance between the two places is $1755 \mathrm{~km}$; (B) The ultrasound image of the right posterior lateral wall showed increased B-lines (large arrow head), localized pleura thickened with irregular border (small arrow), a small atelectasis area (2 large arrows) under the pleura; (C) Chest CT showed irregular stripes in right lung near the pleura, increased patchy densities consolidation shadows, and blurred edge, mainly in the lower lobe of the right lung. Inflatable bronchial shadows was seen in the lesion.

Table 1 Evidence level and recommended strength

Level of evidence recommendation strength

A Strongly recommended: positive evidence-based evidence can improve clinical outcomes, with more benefits than disadvantages

B Recommendation: Good evidence-based evidence can improve clinical outcomes, with more benefits than disadvantages

C Recommendation: Based on expert opinion

D Against recommendation: Based on expert opinion

E Objection to recommendation: Good evidence, not to improve clinical outcomes, or do more harm than good

F Strongly opposed to recommendation: Evidence-based evidence affirms that it does not improve clinical outcomes or does more harm than good

I Not recommended or recommended routinely: Insufficient, lack of evidence or conflicting results of recommendation or objection, pros and cons cannot be assessed

security and ethics could become a big challenge when being transmitted for remotely use. The loss, damage, leakage, and inappropriate use of medical imaging data is unethical and will bring legal issues.

Expert opinion: The content of the clause refers to the actual experience and literature reports of domestic and foreign experts. (Recommended strength: B. Recommendation: Good evidence-based evidence can help ensure the sustainable and standardized development of remote ultrasound.)

\section{Conclusions}

In the course of prevention and control of COVID-19 pandemic, we have learned from the positive feedback of frontline healthcare workers that ultrasound plays an indispensable role in the diagnosis, treatment and follow up of patients with COVID-19. The portable ultrasound can be easily placed in the isolation ward, and the palm ultrasound can be placed in the pocket and carried around. With the support of $4 \mathrm{G}$ or $5 \mathrm{G}$ networks, remote ultrasound can also be used for immersive remote consultations, making it suitable for using in isolated medical areas.
Because SARS-Cov-2 is transmitted by respiratory, contact, and air, the disinfection of the working area as well as the ultrasound machine is of great importance. The above requires the cooperation among radiologist, sonographers, and other hospital staff to promote a safe work environment to minimize the disease spread.

Level of evidence and recommendation strength: Each recommendation from this expert consensus would be supported with corresponding literatures or the clinical practice experience from experts in our writing group. We listed the different levels of strength of recommendations in Table 1 in accordance to the scientific concept of evidence-based medicine.

\section{Acknowledgments}

We wish to thank Xuexia Shan, MD, and Shiyue Zhao, MD, from Hainan Hospital of PLA General Hospital for their assistant. And thank Dr. Yang Hai from Jefferson Thomas Jefferson University for editing and revising the article.

\section{Declaration}

This Expert Consensus was organized by National Health Commission Capacity Building and Continuing Education Center, The War Trauma 
and Emergency Ultrasound Committee and Tele- and Mobile-Ultrasound Subcommittee of Medical Ultrasound Equipment Society of Chinese Association of Medical Equipment.

The contents of this article in Chinese was peer-reviewed and accepted by the journal of Chinese Emergency Medicine for 2020 40(3):185-195. This English version of the consensus was adapted from the Chinese one with permission. The link of Chinese vision of the article as follows: http://111.40.160.75:802/CN/10.3969/j.issn.1002-1949.2020.03.001

\section{Conflict of Interest}

The authors have no conflict of interest to declare.

\section{References}

[1] National Health Commission of the People's Republic of China. Update on pneumonia of novel coronavirus infections as of 24:00 on April 09, 2020. [In Chinese]. Available from: http://2019ncov. chinacdc.cn/2019-nCoV/global.html.

[2] Xu YH, Dong JH, An WM, Lv XY, Yin XP, Zhang JZ, et al. Clinical and computed tomographic imaging features of novel coronavirus pneumonia caused by SARS-CoV-2. J Infect 2020; 80: 394-400.

[3] Xu Z, Shi L, Wang Y, Zhang J, Huang L, Zhang C, et al. Pathological findings of COVID-19 associated with acute respiratory distress syndrome. Lancet Respir Med 2020; 8: 420-422.

[4] Xu X, Yu C, Qu J, Zhang L, Jiang S, Huang D, et al. Imaging and clinical features of patients with 2019 novel coronavirus SARSCoV-2. Eur J Nucl Med Mol Imaging 2020; 47: 1275-1280.

[5] Soldati G, Smargiassi A, Inchingolo R, Buonsenso D, Perrone T, Briganti DF, et al. Is there a role for lung ultrasound during the COVID-19 pandemic? J Ultrasound Med 2020. DOI: 10.1002/jum.

[6] Soldati G, Smargiassi A, Inchingolo R, Buonsenso D, Perrone T, Briganti DF, et al. Proposal for international standardization of the use of lung ultrasound for COVID-19 patients; a simple, quantitative, reproducible method. J Ultrasound Med 2020. DOI: 10.1002/jum.

[7] Kalafat E, Yaprak E, Cinar G, Varli B, Ozisik S, Uzun C, et al. Lung ultrasound and computed tomographic findings in pregnant woman with COVID-19. Ultrasound Obstet Gynecol 2020. DOI: 10.1002/ uog.22034.

[8] Rouby JJ, Arbelot C, Gao Y, Zhang M, Lv J, An Y, et al; APECHO study group. Training for lung ultrasound score measurement in critically ill patients. Am J Respir Crit Care Med 2018. DOI: 10.1164/rccm.201802-0227LE.

[9] Lichtenstein DA, Meziere GA. Relevance of lung ultrasound in the diagnosis of acute respiratory failure: the BLUE protocol. Chest 2008, 134: 117-125.

[10] Lichtenstein D. Lung ultrasound in the critically ill. Curr Opin Crit Care 2014; 20: 315-22.

[11] Lichtenstein DA. BLUE-protocol and FALLS-protocol: two applications of lung ultrasound in the critically ill. Chest 2015; 147: 1659-1670

[12] Pan F, Ye T, Sun P, Gui S, Liang B, Li L, et al. Time course of lung changes on chest ct during recovery from 2019 novel coronavirus (COVID-19) pneumonia. Radiology 2020: 200370. DOI: 10.1148/ radiol.2020200370.

[13] Dietrich CF, Mathis G, Blaivas M, Volpicelli G, Seibel A, Wastl D, et al. Lung B-line artefacts and their use. J Thorac Dis 2016;8:1356-65.

[14] Wang G, Ji X, Xu Y, Xiang X. Lung ultrasound: a promising tool to monitor ventilator-associated pneumonia in critically ill patients. Crit Care 2016; 20: 320.

[15] Inglis AJ, Nalos M, Sue KH, Hruby J, Campbell DM, Braham RM, et al. Bedside lung ultrasound, mobile radiography and physical examination: a comparative analysis of diagnostic tools in the critically ill. Crit Care Resusc 2016; 18: 124.

[16] Huang C, Wang Y, Li X, Ren L, Zhao J, Hu Y, et al. Clinical features of patients infected with 2019 novel coronavirus in Wuhan, China. Lancet 2020; 395: 497-506.

[17] Ludvigsson JF. Systematic review of COVID-19 in children shows milder cases and a better prognosis than adults. Acta Paediatr 2020 DOI: 10.1111/apa.15270.

[18] Su L, Ma X, Yu H, Zhang Z, Bian P, Han Y, et al. The different clinical characteristics of corona virus disease cases between children and their families in China - the character of children with COVID-19. Emerg Microbes Infect 2020; 9: 707-713.

[19] Miller A, Mandeville J. Predicting and measuring fluid responsiveness with echocardiography. Echo Res Pract 2016; 3: G1G12.

[20] Wu CY, Cheng YJ, Liu YJ, Wu TT, Chien CT, Chan KC; NTUH Center of Microcirculation Medical Research (NCMMR). Predicting stroke volume and arterial pressure fluid responsiveness in liver cirrhosis patients using dynamic preload variables: A prospective study of diagnostic accuracy. Eur J Anaesthesiol 2016; 33: 645-52.

[21] Zhang Q, Liu D, Wang X, Zhang H, He H, Chao Y, et al. Inferior vena cava diameter and variability on longitudinal plane measured through ultrasonography from different sites: a comparison study. Chinese Journal Internal Medicine 2014, 53: 880-888. [In Chinese]. DOI: 10.3760/cma.j.issn.0578-1426.2014.11.009

[22] Liang SJ, Tu CY, Chen HJ, Chen CH, Chen W, Shih CM, et al. Application of ultrasound-guided pigtail catheter for drainage of pleural effusions in the ICU. Intensive Care Med 2009; 35: 350-354.

[23] Piton G, Capellier G, Winiszewski H. Ultrasound-guided vessel puncture: calling for Pythagoras' help. Crit Care 2018; 22: 292.

[24] Saugel B, Scheeren TWL, Teboul JL. Ultrasound-guided central venous catheter placement: a structured review and recommendations for clinical practice. Crit Care 2017; 21: 225.

[25] Shrestha GS. Longing for better ultrasound-guided subclavian/ axillary venous cannulation. Crit Care 2018; 22: 148.

[26] Patel B, Chatterjee S, Davignon S, Herlihy JP. Extracorporeal membrane oxygenation as rescue therapy for severe hypoxemic respiratory failure. $J$ Thorac Dis 2019; 11: S1688-S1697.

[27] Bautista-Rodriguez C, Sanchez-de-Toledo J, Da Cruz EM. The role of echocardiography in neonates and pediatric patients on extracorporeal membrane oxygenation. Front Pediatr 2018; 6: 297.

[28] IJsselstijn H, Hunfeld M, Schiller RM, Houmes RJ, Hoskote A, Tibboel D, et al. Improving long-term outcomes after extracorporeal membrane oxygenation: from observational follow-up programs toward risk stratification. Front Pediatr 2018; 6: 177.

[29] Rabie NZ, Sandlin AT, Barber KA, Ounpraseuth S, Nembhard W, Magann EF, et al. Teleultrasound: How Accurate Are We? $J$ Ultrasound Med 2017; 36: 2329-2335. 


\section{Attachment}

\section{Acute and Severe COVID-19 Ultrasound Report}

Ultrasound Record of Lungs and Heart for Critical Illness

\begin{tabular}{|c|c|c|}
\hline Name & ID & Age \\
\hline \multicolumn{3}{|c|}{ 1. Ultrasound examination of lungs } \\
\hline & Right lung & Left lung \\
\hline \multicolumn{3}{|l|}{$\mathrm{N}$} \\
\hline \multicolumn{3}{|l|}{ B1 } \\
\hline \multicolumn{3}{|l|}{ B2 } \\
\hline \multicolumn{3}{|l|}{$\mathrm{C}$} \\
\hline AT & & \\
\hline
\end{tabular}

Gender

Number

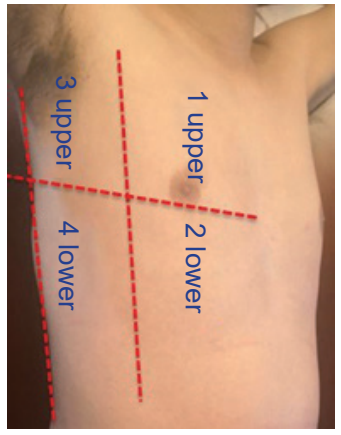

The six-zone of lung ultrasound examination

Notes:

$\mathrm{N}$ : A-line or B-lines are less than 2, which suggest normal.

B1: Multiple B-lines, and the distance between B-lines is about 7mm (B7 line).

B2: Multiple B-lines, and the distance between B-lines is about 3mm (B3 line).

$\mathrm{C}$ : The lung shows acinar, patch, nodules, bronchial signs or fluid bronchial signs with or without a small amount of pleural effusion. These suggest lung consolidation.

AT (Atelectasis): The lesions of lung present hepatic changes, lung volume decrease, a large of pleural effusion. These suggest pulmonary atelectasis.

\section{Echocardiography}

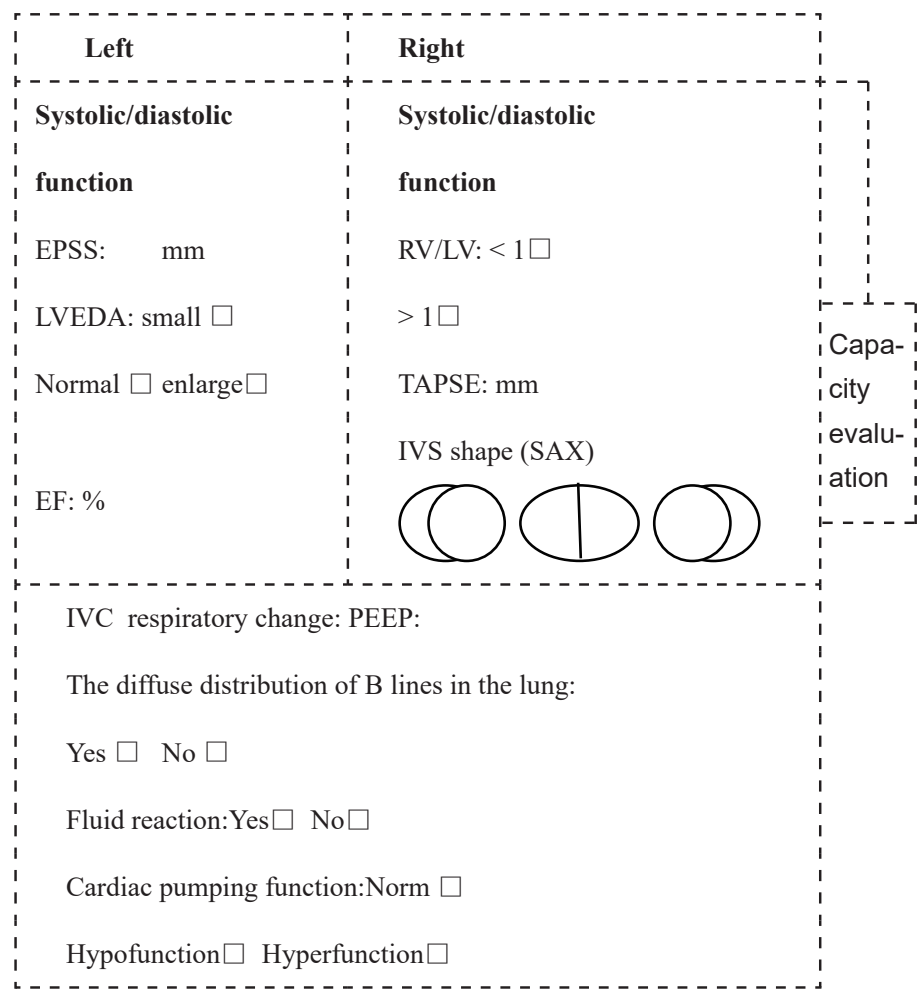

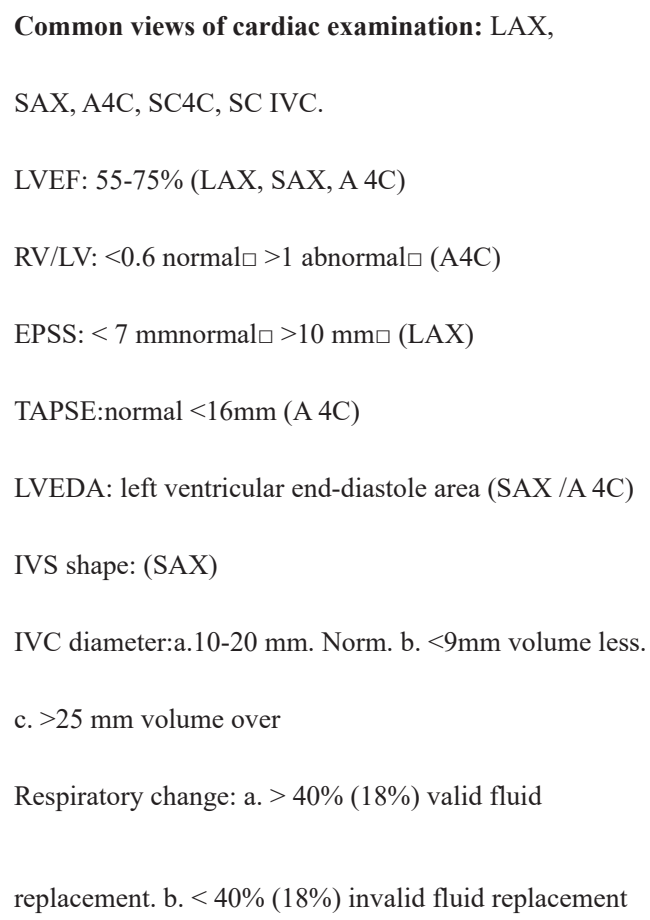

